\title{
Effectiveness of home based early intervention on children's BMI at age 2: randomised controlled trial
}

The authors of this Research paper (BMJ 2012;344:e3732, doi:10.1136/bmj.e3732) have alerted us to an error in the paragraph "Sample size." The second sentence should have read: "To have $80 \%$ power to detect a difference in mean BMI of 0.38 [not " 0.25 "] units between the groups at age 2 at the two sided $5 \%$ significance level, we needed a sample size of 252 per group." The incorrectly published value of 0.25 is, in fact, the calculation for the BMI z score (SD 1.0). BMI z scores were compiled (reported at www.bmj.com/content/344/bmj.e3732/rr/594950) but not stated in the published paper as BMI was identified as the best measure of change in adiposity. In addition, the data collection was all at 24 months; therefore an adjustment of age (with BMI z score) became unnecessary. This correction has no effect on the results.

Cite this as: BMJ 2013;346:f1650

๑ BMJ Publishing Group Ltd 2013 